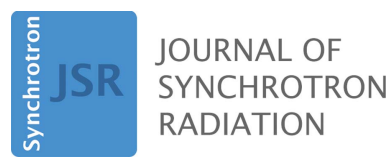

ISSN 1600-5775
Received 7 October 2019

Accepted 22 January 2020

Edited by A. Bergamaschi, Paul Scherrer Institut, Switzerland

We would like to dedicate this article to our colleague and friend Claudio Ferrero, who suddenly passed away in 2018.

Keywords: powder diffraction; geometry calibration; goniometers; moving detectors; translation tables; pair distribution function.

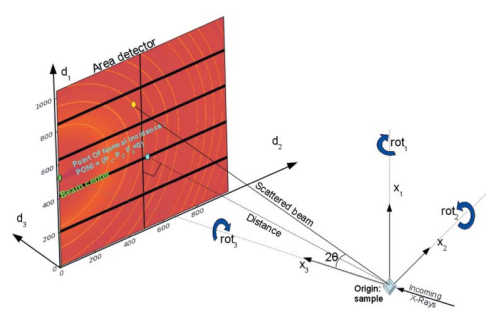

\section{New tools for calibrating diffraction setups}

\author{
J. Kieffer, ${ }^{a} *$ V. Valls, ${ }^{a}$ N. Blanc ${ }^{b, c}$ and C. Hennig ${ }^{d, e}$
}

${ }^{\mathbf{a}}$ ESRF - The European Synchrotron, CS40220, 38043 Grenoble Cedex 9, France, ${ }^{\mathbf{b}}$ University Grenoble Alpes, Grenoble INP, 38000 Grenoble, France, ' CNRS, Institut NEEL, 38000 Grenoble, France, ${ }^{\mathbf{d}}$ Helmholtz-Zentrum Dresden-Rossendorf, Institute of Resource Ecology, Bautzner Landstrasse 400, 01328 Dresden, Germany, and ${ }^{\mathbf{e}}$ The Rossendorf Beamline at ESRF, BP 220, 38043 Grenoble Cedex 9, France. *Correspondence e-mail: jerome.kieffer@esrf.eu

New calibration tools in the $p y F A I$ suite for processing scattering experiments acquired with area detectors are presented. These include a new graphical user interface for calibrating the detector position in a scattering experiment performed with a fixed large area detector, as well as a library to be used in Jupyter notebooks for calibrating the motion of a detector on a goniometer arm (or any other moving table) to perform diffraction experiments.

\section{Introduction}

According to an internal survey of the beamline scientists at the European Synchrotron (ESRF) in 2016, the factor limiting user productivity at diffraction beamlines is the calibration of the experimental setup before the raw data can be reduced to analysable data. The calibration issue is currently worked around by providing users with the proper geometry description or even the reduced data; however, this prevents re-processing at home institutes and also re-interpretation of data, which is needed as a part of the open-data initiative (Wilkinson et al., 2016).

At X-ray facilities such as synchrotons, large area detectors are preferred for gathering the maximum number of photons in scattering experiments (powder diffraction, small-angle scattering, etc.). Using a fixed-position setup combined with the speed of modern detectors (up to a few $\mathrm{kHz}$ ), it is easy to follow chemical reactions or other physical processes in situ. Even with fixed geometries, determining the detector position is still an issue for the majority of users. Hence, a new graphical user interface has been developed for the Python fast azimuthal integration library pyFAI (Kieffer et al., 2019), focusing on the user experience to grant them autonomy in data analysis once they have returned to their home institutes.

Laboratory source diffractometers are commonly equipped with (small) area detectors mounted on moveable goniometer arms for powder diffraction (for example, the Rigaku HyPix3000 detector). Detectors at synchrotrons are often mounted on goniometer or translation tables to provide the degrees of freedom (hereafter DoFs) needed to align the beamline, although those DoFs are rarely used for data acquisition. One counter-example is reported by Gao et al. (2016), where the beamline is equipped with a moving strip-detector, the Mythen detector (1D) from Dectris, but the Mythen is not an area detector.

Pair distribution function (PDF) analysis typically requires very large area detectors and higher energies to be able to cover the needed high $q$-range in one single frame (Chupas et $a l ., 2003)$. When speed is not critical, PDF experiments can be performed with small area detectors mounted on a motorized 
arm, and moved in front of the sample during data acquisition to cover a larger solid angle. A new pyFAI module which handles goniometers and moving detectors is also presented. It offers the ability to exploit the positioning system of the detector (often readily available on beamlines) to acquire powder diffraction or PDF data on a larger $q$-range with no additional cost or investment.

The pyFAI library (Ashiotis et al., 2015) is first briefly introduced, then the merging of multiple diffraction images acquired at different positions is demonstrated, as shown by Kieffer \& Wright (2013). After summarizing how calibration works in $p y F A I$, the new graphical user interface is presented. Finally, the procedure on how to calibrate the absolute position of every single pixel in the detector when mounted on a goniometer (or on a translation stage) as a function of motor positions is described.

\section{The Python fast azimuthal integration library}

pyFAI is a Python (van Rossum, 1989) library used to transform 2D diffraction images into $1 \mathrm{D}$ powder diffraction patterns by re-binning the pixel positions in polar coordinates. The radial units are typically the scattering angle $2 \theta$ or the momentum transfer $q=4 \pi \sin (\theta) / \lambda$. pyFAI also provides additional tools to calibrate the detector position, i.e. to determine its location in space by means of Debye-Scherrer conical rings resulting from the intersection of the diffracted $\mathrm{X}$-ray beam with the detector surface. The observed rings are deformed into ellipses when the detector is planar but slightly inclined. The azimuthal integration is performed in two steps. The first is a pixel-wise transformation corresponding to the image correction,

$$
I_{\text {cor }}=\frac{\text { signal }}{\text { normalization }}=\frac{I_{\text {raw }}-I_{\text {dark }}}{F \Omega P A I_{0}},
$$

where $I_{\text {raw }}$ is the raw signal of the detector, $I_{\text {dark }}$ is the dark current image (it may also be the background image for certain experiments), $F$ is the factor accounting for the flatfield correction, $\Omega$ is the solid angle subtended by a given pixel, $P$ is the polarization correction term and $A$ represents the detector's apparent efficiency due to the incidence angle of the photon on the detector (for integrating detectors, highenergy photons with larger incidence angles see larger sensor thickness, and thus have a higher detection probability). $I_{\text {raw }}$ may be normalized by the incoming flux $I_{0}$, which is independent of the pixel position. The numerator of equation (1) will hereafter be referred to as the 'signal', whilst the denominator will be referred as 'normalization'. The 'variance' associated with the signal of every single pixel can be estimated from statistical distributions assuming, for example, that the detector has a Poissonian behaviour. This variance needs to be scaled in a similar way when considering error propagation.

The second step is the re-binning of the data, which is carried out using histograms of the radial positions, weighted by either the signal or normalization as described by Kieffer \& Ashiotis (2014). This histogram sums the signal (resp. normalization) for all pixels having a radius corresponding to a given radial bin (this set of pixels is denoted $\operatorname{bin}_{\mathrm{r}}$ ). The intensity averaged over all pixels located at a given radial value is then simply the ratio of the two histograms which bin corresponds to the radius of interest [equation (2)]. The propagated error (standard error of the mean) is given in equation (3),

$$
\begin{gathered}
\langle I\rangle_{\mathrm{r}}=\frac{\sum_{i \in \text { bin }_{\mathrm{r}}} c_{i} \cdot \text { signal }_{i}}{\sum_{i \in \text { bin }_{\mathrm{r}}} c_{i} \cdot \text { normalization }_{i}}, \\
\sigma_{\mathrm{r}}(I)=\frac{\sqrt{\sum_{i \in \text { bin }_{\mathrm{r}}} c_{i}^{2} \cdot \text { variance }_{i}}}{\sum_{i \in \text { bin }_{\mathrm{r}}} c_{i} \cdot \text { normalization }_{i}} .
\end{gathered}
$$

In equations (2) and (3), $c_{i}$ is used to describe pixel splitting and corresponds to the fraction of the pixel area falling into the specific histogram bin $\left(0 \leq c_{i} \leq 1\right)$. The multiple pixelsplitting schemes available in $p y F A I$, called full-splitting, bounding-box-splitting and no-splitting, have been described by Ashiotis et al. (2015).

The integration scheme presented in equation (2) is an improvement over former versions of $p y F A I$ (version $<0.18$ ), which used equation (4) where the error propagation was not properly performed as pixels with smaller normalization factors were over-represented in the associated errors,

$$
\langle I\rangle_{\mathrm{r}}=\frac{\sum_{i \in \text { bin }_{\mathrm{r}}}\left(c_{i} \cdot \text { signal }_{i} / \text { normalization }_{i}\right)}{\sum_{i \in \text { bin }_{\mathrm{r}}} c_{i}} .
$$

\section{Azimuthal integration of multiple frames taken at multiple geometries}

The pyFAI integration scheme of multiple images taken at various positions was first reported by Kieffer \& Wright (2013). The procedure is conceptually similar to the integration on a single image, except that all histograms need to be calculated over the very same grid (bin positions) to allow histograms from all images to be summed together, as described in equation (5),

$$
\langle I\rangle_{\mathrm{r}}=\frac{\sum_{\text {imges }} \sum_{i \in \text { bin }_{\mathrm{r}}} c_{i} \cdot \text { signal }_{i}}{\sum_{\text {imges }} \sum_{i \in \text { bin }_{\mathrm{r}}} c_{i} \cdot \text { normalization }_{i}} .
$$

The normalization for solid-angle correction, $\Omega$, has to be performed using an absolute solid-angle reference system as different geometries may have very different sample-todetector distances. This is different from the integration in single-frame mode, where $p y F A I$ uses the solid angle relative to the point of normal incidence (PONI). Hence, integrated intensities in multi-geometry mode are orders of magnitude larger than usual, the scale factor being the solid angle of one pixel at the PONI, i.e. $\operatorname{dist}^{2} /\left(\operatorname{pixel}_{1} \times \operatorname{pixel}_{2}\right)$. Errors are 
propagated in an similar way to equation (3), taking into account the absolute solid-angle normalization.

\section{Calibration of the detector position}

\subsection{Principle of the calibration using} a Debye-Scherrer diffraction image

The calibration of a detector position is performed using several DebyeScherrer rings collected from a reference powder called the calibrant. The rings are extracted automatically from the image (by subtracting a smoothed version of the image) and control points are placed at the local maxima on the rings. The geometry of the experiment is obtained by refining geometric parameters using a leastsquare fitting of the $2 \theta$ values calculated for the different control points.

Intuitively, the easiest geometry to perform the calibration is built upon the beam-centre definition, which is the intersection of the direct beam with the detector. This geometry was first introduced in FIT2D (Hammersley et al., 1996) and re-used in many software packages such as GSAS-II (Toby \& Von Dreele, 2013) and DAWN (Filik et al., 2017) which also offer user-friendly interfaces. The FIT2D geometry reaches its limits when the detector is heavily tilted, and it is not even possible to describe a detector mounted parallel to the beam (which is sometimes used on laboratory sources in reflection geometry). The geometry used in pyFAI is based on the definition of the PONI (Fig. 1), which is the orthogonal projection of the sample position (the origin in $p y F A I$ ) on the detector plane (or the plane $z=d_{3}=0$ when the detector is non-planar and $z$ varies from pixel to pixel). This geometry is inspired by SPD (Boesecke, 2007) and is capable of describing any detector position in space. It is worth noting that the PONI is co-located with the beam centre when the detector is not tilted, i.e. mounted orthogonal to the direct beam. Moreover, the PONI is more likely than the beam centre to be located within the detector surface when the detector is heavily tilted (since the detector gathers more photons when facing the sample). Some geometry conversion tools are provided by $p y F A I$ and documented by Detlefs \& Kieffer (2019).

The geometrical parameters to be refined are the following: (i) dist: the distance (in metres) from the sample position to the PONI. (ii) poni $i_{1}$ and poni $i_{2}$ : space coordinates of the PONI (in metres) within the detector plane $\left(z=d_{3}=0\right)$ along the slow- and fast-reading dimension of the detector image ( 1 and 2 usually refer to the row and the column axis, i.e. $y$ and $x$, respectively). (iii) $\operatorname{rot}_{1}, \operatorname{rot}_{2}$ and $\operatorname{rot}_{3}$ : the rotation angles (expressed in radians) of the detector placed at the proper distance from the sample, with respect to the three axes of the laboratory reference system. The detector is first rotated around the vertical axis $\left(r o t_{1}\right)$, then around the horizontal axis $\left(\mathrm{rot}_{2}\right)$ and finally around the incoming beam direction $\left(\mathrm{rot}_{3}\right)$. In a more mathematical way, this gives

$$
R=R_{3} R_{2} R_{1}
$$

where

$$
\begin{aligned}
& R_{1}=\left[\begin{array}{ccc}
1 & 0 & 0 \\
0 & \cos \left(r o t_{1}\right) & \sin \left(r o t_{1}\right) \\
0 & -\sin \left(r o t_{1}\right) & \cos \left(r o t_{1}\right)
\end{array}\right] \text {, } \\
& R_{2}=\left[\begin{array}{ccc}
\cos \left(r o t_{2}\right) & 0 & \sin \left(r o t_{2}\right) \\
0 & 1 & 0 \\
-\sin \left(r o t_{1}\right) & 0 & \cos \left(r o t_{2}\right)
\end{array}\right] \text {, } \\
& R_{3}=\left[\begin{array}{ccc}
\cos \left(\mathrm{rot}_{3}\right) & -\sin \left(\mathrm{rot}_{3}\right) & 0 \\
\sin \left(\mathrm{rot}_{3}\right) & \cos \left(\mathrm{rot}_{3}\right) & 0 \\
0 & 0 & 1
\end{array}\right] \text {. }
\end{aligned}
$$

It is worth mentioning that rotations $R_{1}$ and $R_{2}$ are lefthanded, while $R_{3}$ is right-handed, which is a legacy from previous versions of $p y F A I$.

The strength of this geometry parameterization is that it describes any detector position in space. The drawback is that some parameters are correlated or not optimized:

(i) dist-wavelength: reducing the wavelength is nearly equivalent to increasing the distance unless the diffraction angle is large $\left(2 \theta>30^{\circ}\right)$. It is advisable to fix one of these two variables unless the data quality are good enough and the scattering range is very large. This is an intrinsic limitation of the single-image approach.

(ii) rot $_{1}$-poni $i_{2}$ and rot $_{2}-$ poni $_{1}$ : as small rotations can be mistaken for larger translations. Fixing one of the two decreases the uncertainty of the other by several orders of 
magnitude. The user interface offers a 'SAXS constrains' button to lock the detector normal to the direct beam.

(iii) rot $_{3}$ : cannot be refined from Debye-Scherrer cones as they are, by definition, invariant by rotation around the incoming beam. The rot $_{3}$ parameter can be assessed using an anisotropic contribution such as the polarization effect or some textures in the sample. This rot $_{3}$ parameter is used to describe the azimuthal rotation of the experimental setup or of the sample, for example, when calculating pole figures.

Those calibration parameters containing the geometry of the experimental setup are saved in text files with a poni extension, also containing the detector definition and the wavelength. Such a file can subsequently be loaded into an azimuthal integrator object, ready to perform the azimuthal regrouping of images coming from the detector.

\subsection{Graphical user interface}

A semi-graphical calibration tool has been available as part of $p y F A I$ since the origin of the project (Ashiotis et al., 2015), but this tool was considered too difficult to use by inexperienced users. Thus, many groups have developed their own graphical user interface on top of $p y F A I$, often optimized for their specific setup or experiment: Dioptas (Prescher \& Prakapenka, 2015) focusing on high-pressure experiments, Dpdak (Benecke et al., 2014) for online SAXS, NanoPeakCell (Coquelle et al., 2015) for serial crystallography, PySAXS (Taché et al., 2015), WiSAXS or XPDFsuite (Yang et al., 2014) to cite a few.

Following the survey conducted among the beamline scientists of the ESRF, a new graphical user interface (GUI) design was developed on top of $p y F A I$. This new GUI, called pyFAI-calib2, is based on the silx scientific widgets (Knobel et al., 2017), itself based on the PyQt5 library (Thompson, 2013). The pyFAI-calib2 tool has been specifically designed to address the needs of novice users, but should perform equally well regardless to the scientific field or experimental setup. The calibration of the experimental setup based on DebyeScherrer rings is carried out in five steps and presented as a software wizard with five subsequent tabs within the graphical interface:

(i) Experimental settings. This first tab lets the user select the wavelength (or the energy), the reference material for calibration (calibrant) and choose the type of detector, either from a list of 50 provided, or by defining a new detector (Fig. 2).

(ii) Mask drawing. This tab allows us to mask-out unwanted regions/pixels from the image, either based on their intensity (thresholding) or simply by drawing polygons on the image. Different masks can be saved, retrieved and assembled (Fig. 3).

(iii) Peak picking. Individual peaks and arcs of rings can be segmented out (automatically) and assigned (manually) to different rings, each associated with a single reflection of the calibrant (Fig. 4).

(iv) Geometry fitting. At this stage, the detector position and wavelength are fitted against peak positions and ring numbers. Any of the parameters can be fixed or let free for
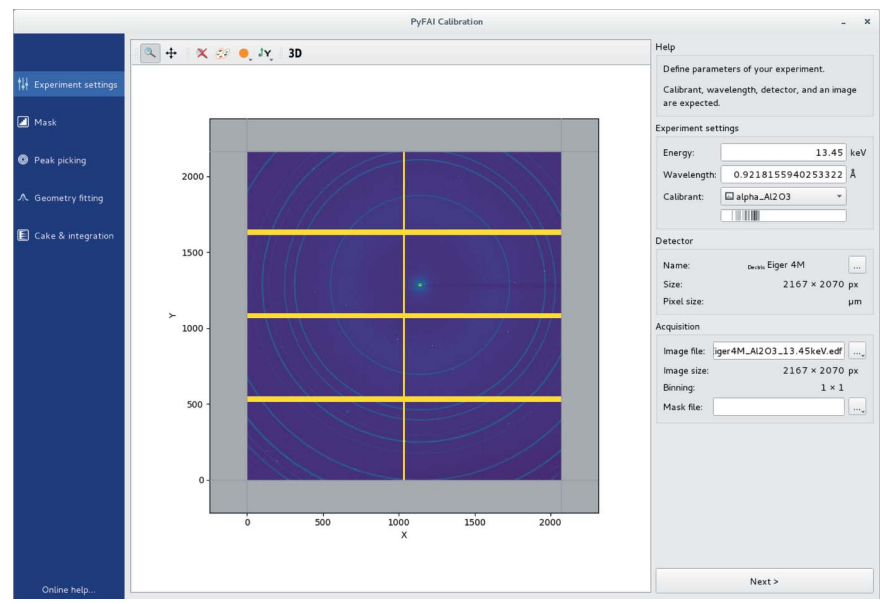

Figure 2

The experiment settings tab is used to load the calibration image, set the energy, the calibrant and select the detector for the subsequent analysis. The binning mode of the detector is automatically guessed.
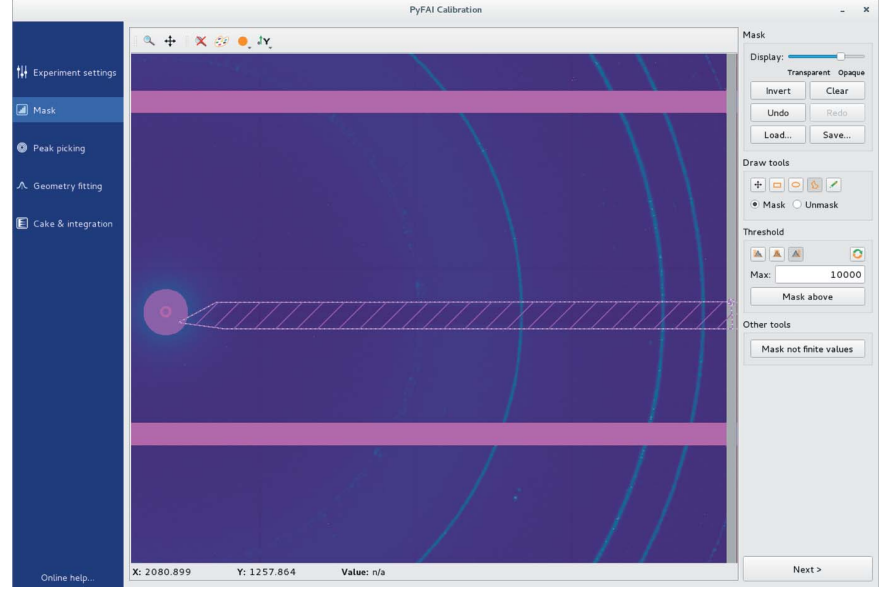

Figure 3

The mask-drawing tool is used to exclude pixels using a rectangular, polygonal or pencil selection. Pixels can also be selected according to their value (thresholding).
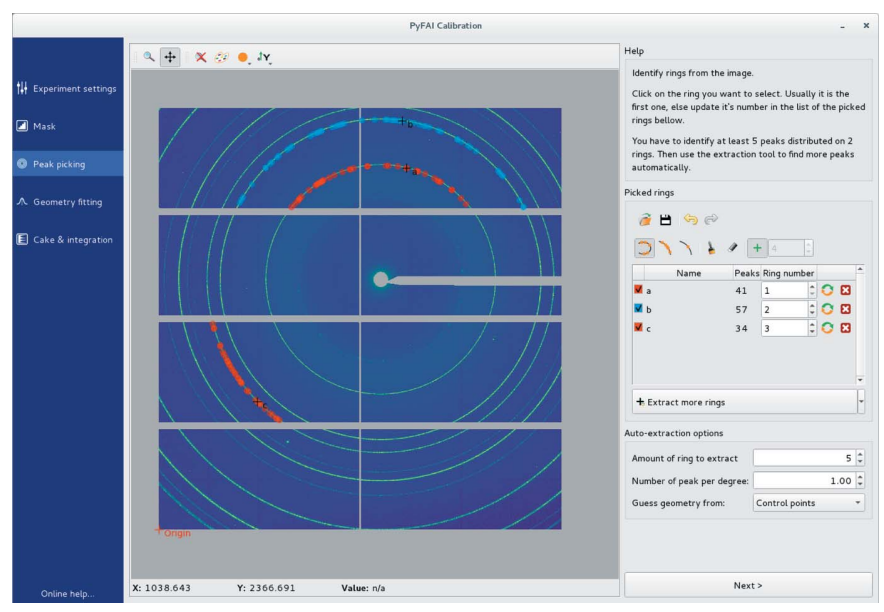

Figure 4

The peak-picking tool automatically selects a group of contiguous local maxima in the image close to the clicked peak which then needs to be assigned to the correct calibrant ring number in the right-hand side panel. 


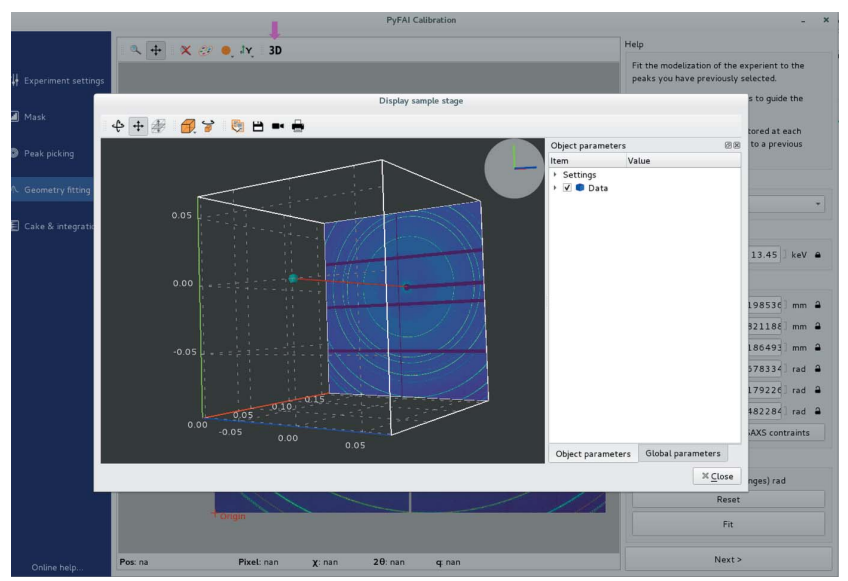

Figure 5

In the geometry fitting tab, each variable can be fixed or left free for refinement within a given range. A $3 \mathrm{D}$ representation of the experimental setup allows visualization of the relative position of the sample, direct beam and detector after fitting.

refinement, possibly with boundaries. The (calculated) positions of the beam centre and the PONI, and the expected positions of rings are overlaid onto the diffraction image to visually assess the quality of the fit. The sample, detector and direct beam can also be visualized in 3D (Fig. 5).

(v) Integration. This tab displays the 1D and 2D integrated patterns (i.e. powder diffraction profile and caked-image) to further validate the modelling of the experimental setup (Fig. 6). The algorithm used for integration, the pixel splitting scheme and the radial unit can also be changed in this tab. Diffraction profiles can be saved as text files or images as well as the experimental setup description file (.poni file) for subsequent use with other tools from the $p y F A I$ suite.

The online documentation of $p y F A I$ contains a quick tutorial (Valls \& Kieffer, 2019) designed for novice users to provide them with their scattering geometry in a couple of minutes. Diffraction images can be provided in HDF5 format (Collette, 2013) or any of the dozens of file formats supported by the FabIO library (Knudsen et al., 2013).
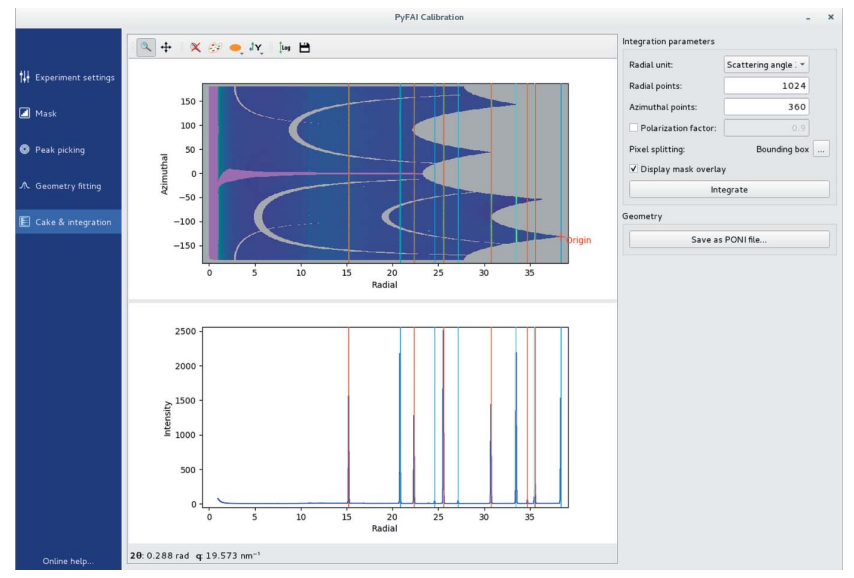

Figure 6

The cake and integration tab displays the $1 \mathrm{D}$ curve and 2D integrated image with the calibrant ring positions overlaid to allow easy validation of the quality of the calibration.

\section{Calibration of a detector on a moving stage}

Calibration of a detector mounted on a translation stage along the direct beam has successfully been exploited by $D A W N$ (Filik et al., 2017) and GSAS-II (Horn et al., 2019) to remove the correlation between the detector distance and the energy of the beam. While these two software packages offer an intuitive user interface for those experiments, the modelization of the translation stage remains hard-coded. This section explains how to describe, programmatically, a moving stage in pyFAI, calibrate the proposed model and use it to perform the azimuthal integration with many images.

The calibration of the detector position on a fixed goniometer position can be performed with $p y F A I$ as long as several rings of the chosen calibrant are present in the image and that five control points can be extracted from one ring and at least one point from another ring. Of course, more points provide a better fit but this limit of two visible rings may be an issue when very small area detectors are used (e.g. the ImXPAD S10).

\subsection{Transformation of geometry}

Here, the difficulty is not in the calibration, but rather in the variety of goniometers and translation stages which may be controlled by one or multiple motors. The goniometer description implemented in pyFAI accepts an arbitrary number of motors attached to the goniometer/translation stage. Let motors be a list of $n$ motors moved during the acquisition: (motor ${ }_{1}$, motor $_{2}, \ldots$, motor $_{n}$ ).

Calibration of the goniometer involves defining a model and optimizing it:

(i) Choose a list of $m$ static parameters for the model which are used to describe the position of the detector, $m$ being the number of DoFs of the model: params $=\left(\right.$ param $_{1}$, param $_{2}, \ldots$, param $_{m}$ ).

(ii) Define the six transformation functions $\Re$ which transform the motor positions (motors) and goniometer parameters (params) into the six PONI parameters used to initialize an azimuthal integrator in $P y F A I$ :

$$
P_{\text {pyFAI }}=\left(\text { dist }_{\text {poni }}, \text { poni }_{2}, \text { rot }_{1}, \text { rot }_{2}, \text { rot }_{3}\right)=\Re_{\text {params }}(\text { motors }) .
$$

(iii) Optimize the parameter set of the goniometer (params) so that, for any position of the motors, the detector position is expressed as a PONI-parameters set.

The six PONI parameters returned are then used to calculate the experimentally observed $2 \theta_{\exp }$ position of the peaks over all control points. Those $2 \theta_{\exp }$ are compared with the theoretically expected $2 \theta_{\text {theo }}$ values calculated from the calibrant $d$-spacing and the wavelength,

$$
\Delta 2 \theta=2 \theta_{\text {exp }}-2 \theta_{\text {theo }}=\arctan \left[\frac{r\left(P_{p y F A I}\right)}{d\left(P_{p y F A I}\right)}\right]-2 \arcsin \left(\frac{\lambda}{2 d}\right) .
$$

The average of the squares of these differences is used as a cost function to optimize the parameters using the scipy. optimize.minimize function from SciPy (Jones et al., 2001), 


$$
\text { CostFunct }=\frac{\sum_{i \in \mathrm{CtIPts}}\left(\Delta 2 \theta_{i}\right)^{2}}{\operatorname{count}(\mathrm{CtlPts})} .
$$

From a computer engineering perspective, these transformation functions present several contradictory challenges: the user should be able to express any numerical transformation (flexibilty), but should be prevented from executing any arbitrary code (security). Moreover, those functions should be made persistable on disk (i.e. saved) and their restoration should be possible without having to re-perform the calibration, nor creating vulnerabilities.

The NumExpr library (Cooke et al., 2009-2017) allows the evaluation of textual mathematical formulae into numerical functions, offering an arbitrary number of parameters for the goniometer definition and as many motors as needed. NumExpr, being a mathematical compiler, cannot execute other types of code. The mathematical expressions provided by the user are simply saved together with the motor names and parameter names and values in a JSON-format (Crockford, 2017) on the disk.

In the following example, the definition of those functions is simply the creation of an object from the GeometryTransformation class (i.e. instantiation), with a list of motor names (pos_names), parameter names (param_names), as well as a set of six formulae, one for each of the six PONI parameters.

\subsection{A simple example: the translation table}

At the ESRF, the protein crystallography beamlines use large area pixel detectors (typically Pilatus 6M from Dectris) placed on a translation table which allows collection of the data at the optimal distance: shorter sample-to-detector distances to explore the region for high- $q$ or longer distances for better Bragg peak separation.

The $M X$-calibrate tool from the $p y F A I$ suite is available for the calibration of many images taken at various distances. This can also be interpreted in terms of the goniometer setup (actually a translation stage), where the sample-to-detector distance is modelled as a simple linear function of the table position:

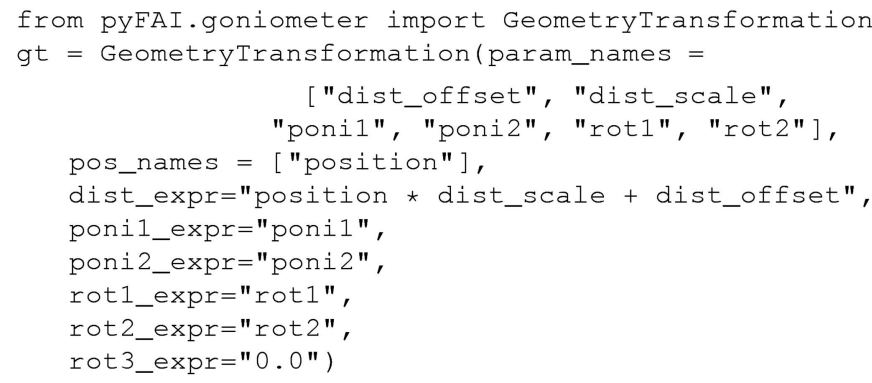

In the example above, a six-parameter model was chosen for the goniometer geometry, most parameters matching exactly those of pyFAI: poni $i_{1}$, poni $i_{2}$, rot $_{1}$ and rot $_{2}$. rot ${ }_{3}$ is forced to zero while the distance is defined as a linear function of the motor position.
The content of this tutorial is available as a Jupyter notebook (Pérez \& Granger, 2007) and forms part of the official documentation of pyFAI (Kieffer \& Flot, 2017); it is directly visible in a web-browser. In addition, one can replay the notebook with Jupyter as it is self-consistent: all images are automatically downloaded and cells can be modified or adapted. The notebook presents the usage of the geometry transformation class together with the translation table refinement. Initially, this set of data was calibrated using the 'MX-calibrate' tool which automatically extracts the control points from images taken at the ESRF ID29 beamline (energy $12.75 \mathrm{keV}$ ) from the metadata written in the image headers, thus a set of control points is available prior to data processing. The translation table is calibrated using all control points and this geometry transformation object. As a result, all images have been integrated azimuthally. Fig. 7 shows the first two Bragg peaks of $\mathrm{CeO}_{2}$ calibrated using this methodology. The blue curve corresponds to this initial model refined; the two peaks look 'doubled', indicating a poor modelling of the geometry.

To address this poor modelling, another transformation function is defined, with a few additional DoFs on the PONI position (hypothesis: the translation table is not perfectly parallel to the incident beam). Once three parameters (dist, poni $i_{1}$ and poni $_{2}$ ) have been re-fitted and all data integrated with the new model, one obtains the orange curve in Fig. 7. Its peaks are much sharper and the residual cost is about five times smaller, indicating a much better fit.

This example shows that the PONI is moving on the detector plane by $1 \%$ o horizontally and $4 \%$ vertically. This 'large' vertical deviation has been confirmed by the beamline staff and is related to the last focusing mirror placed just before the sample, causing the beam to deflect away from the horizontal. Once everything is fitted, the quality of the geometry obtained is perfectly suited to powder diffraction experiments for any detector position.

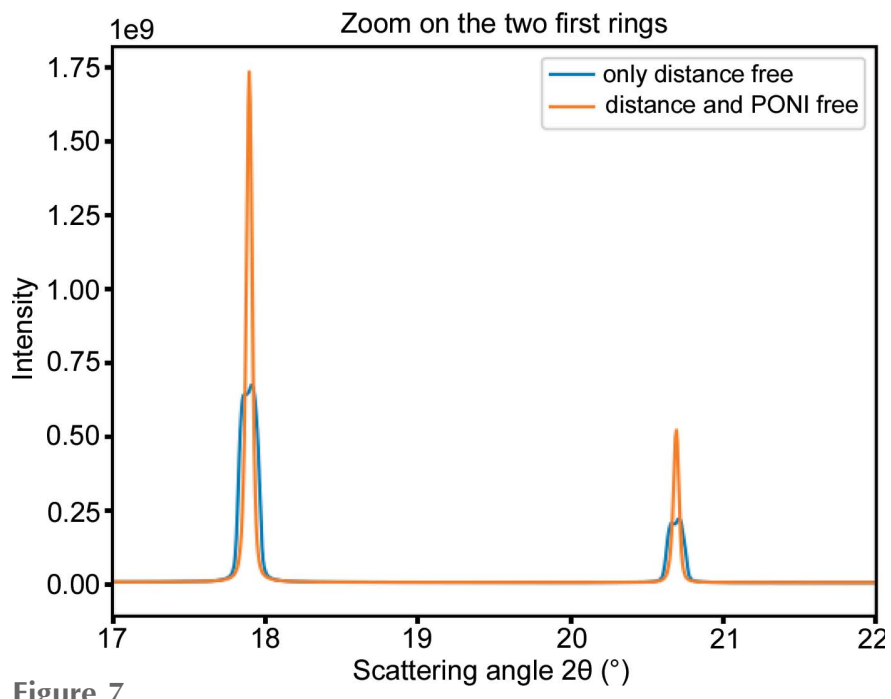

Figure 7

Powder diffraction profile obtained from seven images acquired at various distances from $15 \mathrm{~cm}$ to $45 \mathrm{~cm}$. The translation table position is combined with six (resp. eight) parameters model-fitted, where dist (resp. dist, poni $i_{1}$ and poni $_{2}$ ) depends linearly on the table position. 
In this example, all motor positions used for calibration were within the accessible range, hence positions have been interpolated. The next example will validate that extrapolation outside the calibration range is also possible.

\subsection{A more realistic example: the single-axis goniometer}

Probably the main application of this work is to place a small area detector on a goniometer $2 \theta$ arm in replacement of a punctual detector (e.g. diode) for powder diffraction measurements. The initial idea was to use a small region of interest (ROI) in the centre of an area detector, and check whether it was possible to rebuild a powder diffraction pattern when moving the detector at various goniometer angles. This ROI has to be of limited size in the dimension orthogonal to the ring and as extended as possible in the tangential direction, with the limit of the curvature of the ring (especially at low $2 \theta$ angles). We considered an ROI 10 pixels wide within a Pilatus $100 \mathrm{k}$ (487 pixels $\times 195$ pixels) which means using only $2 \%$ of the total width of the detector (different lines being used for different bins). By using the full detector area, the signal/noise ratio could be improved by a factor of seven $\left[(487 / 10)^{1 / 2}\right]$ if the movement of the detector on the goniometer is perfectly predictable and reproducible.

When the detector arm is moving in the vertical plane (around an horizontal axis), a simple geometry transformation is defined with rot $_{2}$ (in radians) as a linear transformation of the motor position (pos is the default motor name), here measured in degrees by the goniometer. The scale parameter, scale $=\pi / 180$, is used to convert angular units. The other parameters dist, poni $i_{1}$, poni $i_{2}$ and rot $_{1}$ are directly mapped to pyFAI's parameters. As done previously, rot $_{3}$ is kept fixed at zero:

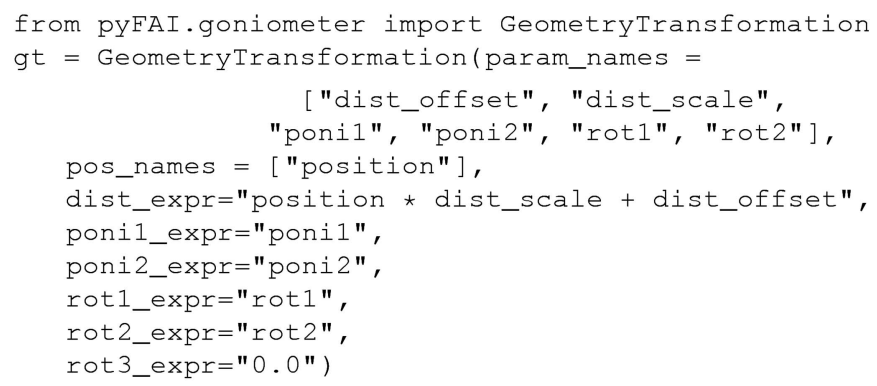

This example is also available in a Jupyter notebook (Kieffer \& Hennig, 2017) and the workflow used is depicted in Fig. 8. This experiment has been performed using a single-module Pilatus (100k) detector which is mounted on a $2 \theta$ arm, moving from $5^{\circ}$ to $65^{\circ}$ in $0.5^{\circ}$ steps on the ROBL beamline (Kvashnina \& Scheinost, 2016), ESRF BM20. In this experiment, 121 diffraction images of $\mathrm{LaB}_{6}$ reference compound material were acquired. Due to the small size of the detector, some of the images present no rings at all (especially at low $2 \theta$ angles), most have only one ring and only a few images display two rings. Even if those images are technically suitable for static detector calibration, they still present a challenge due to the large diffraction angles $\left(2 \theta>30^{\circ}\right)$, the low curvature of the

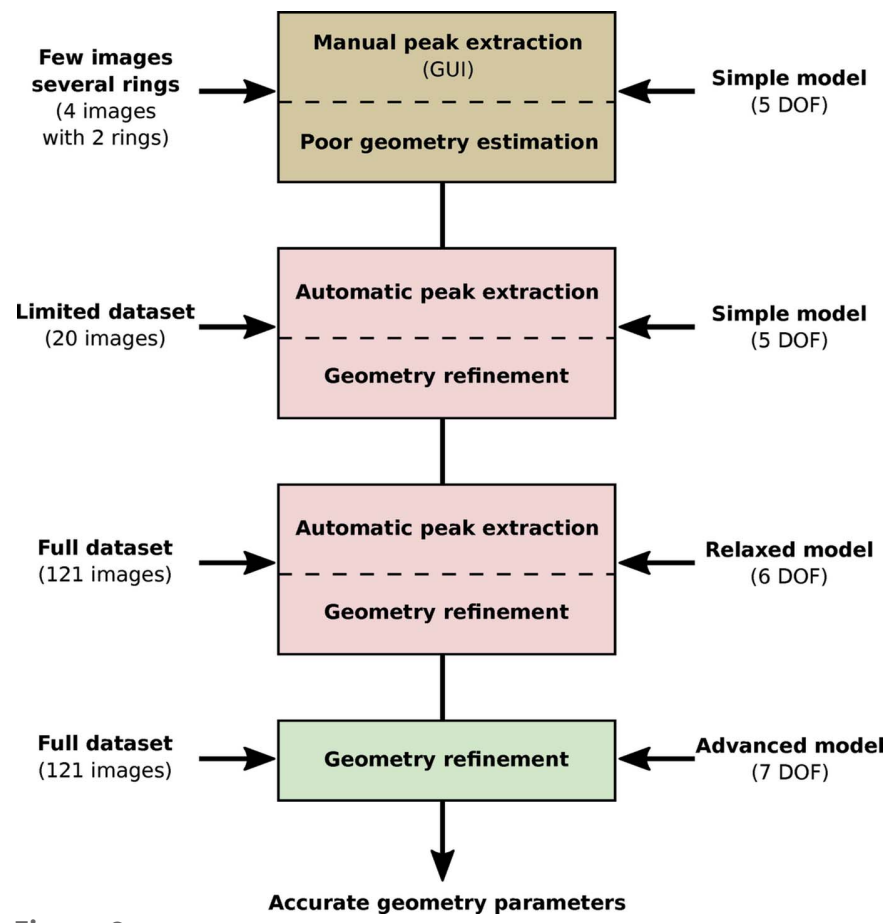

Figure 8

Workflow used to calibrate a set of 121 images collected with a Pilatus $100 \mathrm{k}$ mounted on a moving $2 \theta$ arm and detailed in the Jupyter Notebook given by Kieffer \& Hennig (2017).

rings and the difficulty in assigning the picked peaks with the proper calibrant ring number.

Four images have been manually calibrated, fixing the vertical rotation axis of the detector $\left(\right.$ rot $\left._{1}=0\right)$ to guarantee some consistency between the calibrations.

An initial simple model with a set of parameters where rot $_{2}$ is equal to the goniometer angle has first led to convergence with some constraints and bounds. In a second step, 20 images in the vicinity of the first set were added to the model, their peaks extracted according to the initial model and the model refined using the control points from the 20 images. Then, all other images were added to the model, and additional control points were automatically extracted from all images according to the previous geometry model. Finally, all constraints and bounds were removed, and the model was refined again and used to generate a MultiGeometry object, which is suitable for integrating many images together. After integration of all 121 images, the powder diffraction pattern displayed in Fig. 9 is obtained (orange curve). All peaks of the curve appear at the correct scattering angle but the first few peaks look exceedingly broad.

This broadening is confirmed by looking at the first ring's image, where the goniometer angle was set to $10^{\circ}$ (Fig. 10). The expected position of the ring (dashed red line) does not properly correspond to the actual ring (yellow on the image). The control points extracted and used in the fitting are plotted in blue.

The fitting procedure averaged the rot $_{1}$ value (rotation around the vertical axis) over all images, but Fig. 10, which is taken on the first ring, suggests this value is slightly wrong. The images of the last rings (visible in the notebook) indicate a 


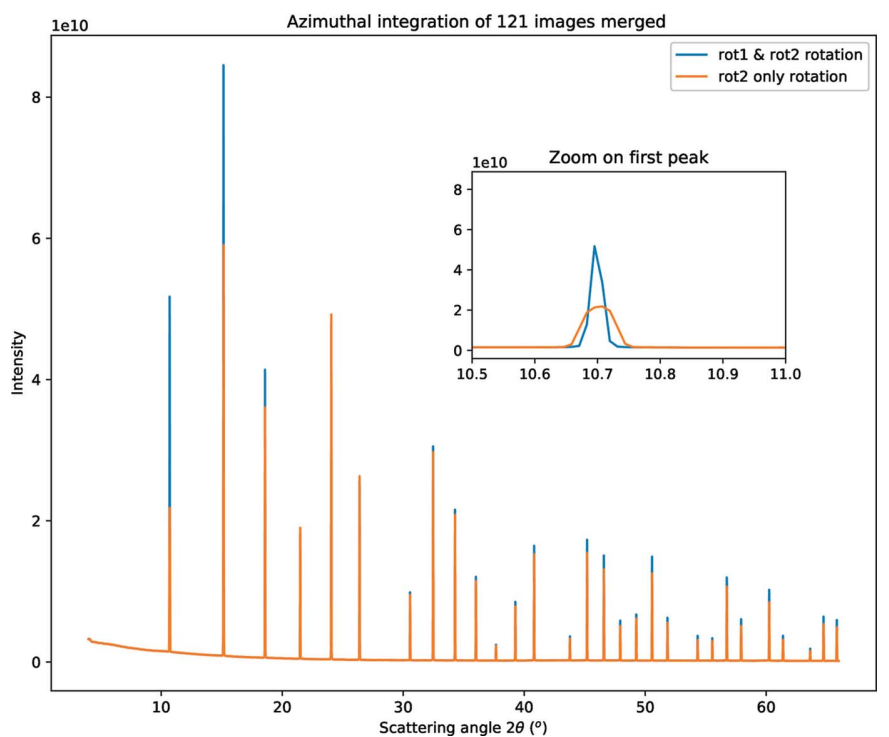

Figure 9

Powder diffraction pattern obtained from 121 Pilatus 100k images acquired at goniometer angles ranging from $5^{\circ}$ to $65^{\circ}$ on an LaB6 reference sample at $16 \mathrm{keV}$. The insert is a close-up view of the first peak showing the sharpness of the signal depending on the model. The orange curve corresponds to the simple model where rot $_{2}$ depends linearly on the goniometer angle (six DoFs). The blue curve corresponds to an advanced model where both rotations (rot ${ }_{1}$ and $\left.r o t_{2}\right)$ depend linearly on the goniometer angle (seven DoFs).

small offset but in the other direction. The fact that both shifts are small suggests to use a first-order correction on rot $_{1}$. A new model was tested where both the $\operatorname{rot}_{1}$ and $\operatorname{rot}_{2}$ values are scaled with the motor position. After refinement, the cost function dropped by a factor two and the low-angle peaks became sharp (blue curve in Fig. 9).

This parameter set was saved and allowed a few other compounds to be analysed and compared with the same pattern recorded with the Pilatus being considered as a point detector. The signal/noise ratio was found to be much better with an acquisition time 24 times faster (10 min instead of $4 \mathrm{~h}$ ). The average peak resolution full width at half-maximum FWHM $=0.02^{\circ}$ obtained on $\mathrm{LaB}_{6}$ (see notebook) is a factor of ten worse than the highest achieved resolution with secondary monochromator on insertion devices (Dejoie et al., 2018), but such an experiment requires $24 \mathrm{~h}$ acquisition.

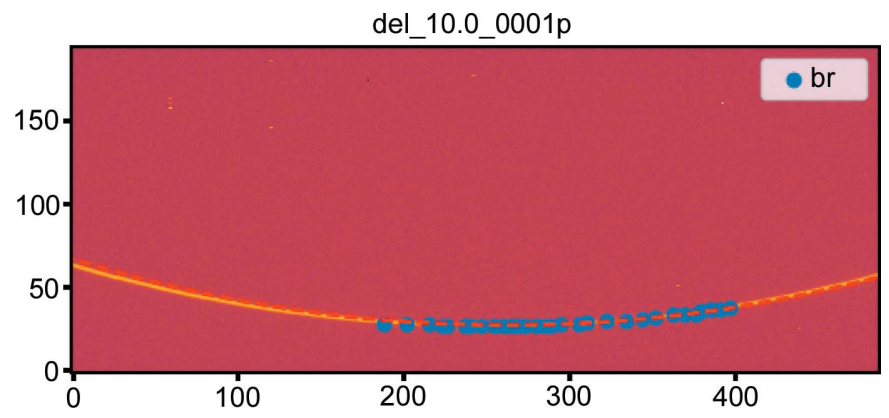

Figure 10

Diffraction image taken with the goniometer arm at $10^{\circ}$. The control points are in blue and the expected ring of the simple model $\left[\mathrm{rot}_{2}=\right.$ $f(p o s)]$ is the dashed red line. This highlights the need for rot $_{1}$ to depend on the goniometer position.
A third example of goniometer calibration is available in the work by Kieffer \& Blanc (2017). It corresponds to the calibration of an ImXPAD detector (Boudet et al., 2003) composed of eight stripes of seven modules, many of which are defective. This detector is mounted on the goniometer arm at the D2AM beamline (Ferrer et al., 1998), ESRF BM02. This example is conceptually the same as that from the ROBL beamline, with a few differences: (i) all images are fitted directly with eight DoFs; (ii) the detector is larger, hence the calculation time is longer, especially when it comes to ring extraction; (iii) the mask needs some extra care to remove a few hot pixels; the detector is mounted, rotated by $90^{\circ}$ on the arm, thus $\operatorname{rot}_{3}=\pi / 2$.

This generic method has even been extended to strip detectors like the Mythen detector manufactured by Dectris as shown in the tutorial on the calibration of the array of nine Mythen detectors (Kieffer \& Picca, 2018) mounted on the goniometer arm of beamline Cristal at Synchrotron Soleil.

\section{Outlook}

The goniometer description in this work can be adapted to many types of goniometers. The transformation function class presented in this manuscript may be extended in the future to use libhkl (Picca, 2010-2016), which already contains many diffractometer geometries with their associated rotation matrices.

Different generations of pixel detectors have seen their pixel sizes shrinking: from $172 \mu \mathrm{m}$ for Pilatus, $130 \mu \mathrm{m}$ for ImXPAD, $75 \mu \mathrm{m}$ for Eiger and $55 \mu \mathrm{m}$ for Medipix-based chips, and probably even less for future-generation detectors dedicated to coherent diffraction imaging. As the resolution of the powder diffraction pattern obtained is often limited by the pixel size (and the sample-to-detector distance), this shrinkage of pixel sizes naturally leads to higher quality powder diffraction patterns. Unfortunately, to keep the covered $2 \theta$ range constant, one would need to multiply the number of pixels by the square of the pixel size reduction factor, and the associated infrastructure for read-out and data transfer accordingly. Moving the detector offers a flexibility which removes this limitation but makes the experiment slightly slower - though still suitable for in situ experiments.

\section{Conclusions}

The new graphical user interface of $p y F A I$ has been developed to ease the calibration of an experimental setup with static detectors, especially for novice users. The concept of calibration of the detector position has been extended to fit the detector position as a function of the motion of a goniometer. Once a few fixed positions of the goniometer have been calibrated, a model can be optimized and the detector position can be extrapolated at any goniometer configuration. By acquiring multiple images at various positions, these images can be integrated together to produce a high- $q$ powder diffraction pattern of quality equivalent to that acquired with a much larger detector, opening up new opportunities for 
in situ experiments and PDF measurements on most synchrotron diffraction beamlines.

\section{Acknowledgements}

We would like to thank all ESRF beamline teams for supporting the $p y F A I$ development, and especially David Flot from the MX-group who provided the data of the translation table. We would also like to thank the other participants in the project, especially Serge Cohen from the Ipanema institute, the DiffAbs and Cristal beamlines and Frédéric Emmanuel Picca from Synchrotron Soleil. In the instrumentation services and development division (ISDD) of the ESRF we would like to thank V. Armando Solé, head of the data analysis unit and leader of the silx project, and all our colleagues from the silx project: Thomas Vincent, Henri Payno, Damien Naudet and Pierre Knobel for their support and ideas. A great thank you to Carsten Detlefs for his contribution to the documentation of the geometry in $p y F A I$.

\section{Funding information}

We would like to thank the French CNRS for financing the IRDRX project in 2015 and 2016, which acted as a catalyst for the goniometer refinement.

\section{References}

Ashiotis, G., Deschildre, A., Nawaz, Z., Wright, J. P., Karkoulis, D., Picca, F. E. \& Kieffer, J. (2015). J. Appl. Cryst. 48, 510-519.

Benecke, G., Wagermaier, W., Li, C., Schwartzkopf, M., Flucke, G., Hoerth, R., Zizak, I., Burghammer, M., Metwalli, E., MüllerBuschbaum, P., Trebbin, M., Förster, S., Paris, O., Roth, S. V. \& Fratzl, P. (2014). J. Appl. Cryst. 47, 1797-1803.

Boesecke, P. (2007). J. Appl. Cryst. 40, s423-s427.

Boudet, N., Berar, J.-F., Blanquart, L., Breugon, P., Caillot, B., Clemens, J.-C., Koudobine, I., Delpierre, P., Mouget, C., Potheau, R. \& Valin, I. (2003). Nucl. Instrum. Methods Phys. Res. A, 510, 4144.

Chupas, P. J., Qiu, X., Hanson, J. C., Lee, P. L., Grey, C. P. \& Billinge, S. J. L. (2003). J. Appl. Cryst. 36, 1342-1347.

Collette, A. (2013). Python and HDF5. Sebastopol, CA: O'Reilly.

Cooke, D., Hochberg, T. \& Alted, F. (2009-2017). NumExpr: Fast Numerical Expression Evaluator for NumPy, https://github.com/ pydata/numexpr.

Coquelle, N., Brewster, A. S., Kapp, U., Shilova, A., Weinhausen, B., Burghammer, M. \& Colletier, J.-P. (2015). Acta Cryst. D71, 11841196.

Crockford, D. (2017). The JSON Data Interchange Syntax, Ecma International, https:/www.ecma-international.org/publications/ standards/Ecma-404.htm.

Dejoie, C., Coduri, M., Petitdemange, S., Giacobbe, C., Covacci, E., Grimaldi, O., Autran, P.-O., Mogodi, M. W., Šišak Jung, D. \& Fitch, A. N. (2018). J. Appl. Cryst. 51, 1721-1733.

Detlefs, C. \& Kieffer, J. (2019). Geometry Transformation: the Example of pyFAI and imaged11, http://www.silx.org/doc/pyFAI/ latest/geometry_conversion.html.

Ferrer, J.-L., Simon, J.-P., Bérar, J.-F., Caillot, B., Fanchon, E., Kaïkati, O., Arnaud, S., Guidotti, M., Pirocchi, M. \& Roth, M. (1998). J. Synchrotron Rad. 5, 1346-1356.

Filik, J., Ashton, A. W., Chang, P. C. Y., Chater, P. A., Day, S. J., Drakopoulos, M., Gerring, M. W., Hart, M. L., Magdysyuk, O. V., Michalik, S., Smith, A., Tang, C. C., Terrill, N. J., Wharmby, M. T. \& Wilhelm, H. (2017). J. Appl. Cryst. 50, 959-966.
Gao, M., Gu, Y., Li, L., Gong, Z., Gao, X. \& Wen, W. (2016). J. Appl. Cryst. 49, 1182-1189.

Hammersley, A. P., Svensson, S. O., Hanfland, M., Fitch, A. N. \& Hausermann, D. (1996). High. Press. Res. 14, 235-248.

Horn, C., Ginell, K. M., Von Dreele, R. B., Yakovenko, A. A. \& Toby, B. H. (2019). J. Synchrotron Rad. 26, 1924-1928.

Jones, E., Oliphant, T. E. \& Peterson, P. (2001). SciPy: Open Source Scientific Tools for Python, http://www.scipy.org/.

Kieffer, J. \& Ashiotis, G. (2014). PyFAI: a Python Library for High Performance Azimuthal Integration on GPU, http://arxiv.org/abs/ 1412.6367.

Kieffer, J. \& Blanc, N. (2017). ImXPAD S540 Detector at D2AM, https://github.com/silx-kit/pyFAI/blob/master/doc/source/usage/ tutorial/Goniometer/Rotation-XPADS540/D2AM-15.ipynb.

Kieffer, J. \& Flot, D. (2017). Calibration of a Detector on a Translation Table, https://github.com/silx-kit/pyFAI/blob/master/ doc/source/usage/tutorial/Goniometer/Translation-Pilatus6M/TT calibration.ipynb.

Kieffer, J. \& Hennig, C. (2017). Calibration of a 2Theta Arm with a Pilatus 100k Detector, https://github.com/silx-kit/pyFAI/blob/ master/doc/source/usage/tutorial/Goniometer/Rotation-Pilatus 100k/Multi120_Pilatus100k.ipynb.

Kieffer, J. \& Picca, F.-E. (2018). Cristal_Mythen.ipynb, https:// github.com/silx-kit/pyFAI/commits/master/doc/source/usage/ tutorial/Soleil/Cristal_Mythen.ipynb.

Kieffer, J., Valls, V., Vincent, T., Wright, J. P., Pandolfi, R., Ashiotis, G., Faure, B., Wright, C. J., Plaswig, F., Neher, S., Flucke, G. \& Hov, A. (2019). silx-kit/pyFAI: PyFAI v0.19, https://doi.org/ 10.5281/zenodo.832896.

Kieffer, J. \& Wright, J. (2013). Powder Diffr. 28, S339-S350.

Knobel, P., Valls, V., Kieffer, J., Solé, V. A., Naudet, D., Payno, H., Retegan, M., Nemoz, C. \& Paleo, P. (2017). silx-kit/silx: v0.5.0, https://doi.org/10.5281/zenodo.576042.

Knudsen, E. B., Sørensen, H. O., Wright, J. P., Goret, G. \& Kieffer, J. (2013). J. Appl. Cryst. 46, 537-539.

Kvashnina, K. O. \& Scheinost, A. C. (2016). J. Synchrotron Rad. 23, 836-841.

Pérez, F. \& Granger, B. E. (2007). Comput. Sci. Eng. 9, 21-29.

Picca, F. E. (2010-2016). libhkl: Diffractometer Computation Control Library, https://packages.debian.org/sid/libhkl5.

Prescher, C. \& Prakapenka, V. B. (2015). High. Press. Res. 35, $223-$ 230.

Rossum, G. van (1989). Python Programming Language, http:// www.python.org.

Taché, O., Thill, A., Spalla, O., Carriere, D., Sen, D., Testard, F. \& Morat, A. (2015). PySAXS: Python Package for Small Angle X-ray Scattering (SAXS) Data Treatment, http://iramis.cea.fr/en/Phocea/ Vie_des_labos/Ast/ast_sstechnique.php?id_ast=1799.

Thompson, P. (2013). PyQt: Whitepaper, https://www.riverbank computing.com/software/pyqt/whitepaper/index.html.

Toby, B. H. \& Von Dreele, R. B. (2013). J. Appl. Cryst. 46, 544-549.

Valls, V. \& Kieffer, J. (2019). Calibration of a Diffraction Setup Using the Graphical User Interface (GUI), http://www.silx.org/doc/pyFAI/ latest/usage/cookbook/calib-gui/index.html.

Wilkinson, M. D., Dumontier, M., Aalbersberg, I. J., Appleton, G., Axton, M., Baak, A., Blomberg, N., Boiten, J.., da Silva Santos, L. B., Bourne, P. E., Bouwman, J., Brookes, A. J., Clark, T., Crosas, M., Dillo, I., Dumon, O., Edmunds, S., Evelo, C. T., Finkers, R., Gonzalez-Beltran, A., Gray, A. J. G., Groth, P., Goble, C., Grethe, J. S., Heringa, J., t Hoen, P. A. C., Hooft, R., Kuhn, T., Kok, R., Kok, J., Lusher, S. J., Martone, M. E., Mons, A., Packer, A. L., Persson, B., Rocca-Serra, P., Roos, M., van Schaik, R., Sansone, S.., Schultes, E., Sengstag, T., Slater, T., Strawn, G., Swertz, M. A., Thompson, M., van der Lei, J., van Mulligen, E., Velterop, J., Waagmeester, A., Wittenburg, P., Wolstencroft, K., Zhao, J. \& Mons, B. (2016). Sci. Data, 3, 160018.

Yang, X., Juhas, P., Farrow, C. L. \& Billinge, S. J. (2014). arXiv: 1402.3163 . 